\title{
DATA COMPLETION FOR LINEAR SYMMETRIC OPERATORS AS A CAUCHY PROBLEM: AN EFFICIENT METHOD VIA ENERGY-LIKE ERROR MINIMIZATION
}

\author{
Thouraya N. Baranger ${ }^{1}$ and Stéphane Andrieux ${ }^{2}$ \\ ${ }^{1}$ Université de Lyon, CNRS \\ LaMCoS, UMR5259, INSA-Lyon, F-69621, Villeurbanne; \\ Université Lyon 1, F-69622, Villeurbanne, France \\ ${ }^{2}$ Mechanics of Sustainable Industrial Structures Laboratory, \\ UMR CNRS-EDF 2832, Clamart, France
}

\begin{abstract}
Data completion is a problem in which known or measured superabundant data exist for part of the boundaries of a domain, whereas the data for the rest of the boundaries are unknown. Thus the aim is to determine the solution of a known PDE defined throughout the domain, which satisfies the superabundant data and then identifies the missing ones. For linear symmetric operators, we propose a general method to solve the data completion problem as a Cauchy problem. Various applications are described for stationary conduction and elastostatic problems.
\end{abstract}

\section{INTRODUCTION}

Given the growth of development in measurement and data imaging technologies [11.], data completion problems can arise in a large range of applications. These problems occur when dealing with PDE known to hold true in a solid for which data is lacking on a part of its boundary but with superabundant boundary data on another part of it. Thus data completion problems consist in recovering the lacking data. It is an inverse, then an ill-posed problem. Examples can be found in thermal and electric conduction problems or linear elasticity, in saturated porous media, for linear fracture mechanics applications.

The data completion problem has been addressed by many authors, firstly for the Laplace operator and mainly in two dimensions. Various approaches have been proposed using boundary element techniques [2,3], fundamental solutions [4,5], regularized least squares methods [6], moment methods associated with the Backus-Gilbert procedure [7], fixed point techniques [8] and, more recently, variational approaches based on SteklovPoincaré operators $[9,10]$.

In this paper, a general method is proposed for linear symmetric operators. It gives a comprehensive and compact presentation of previous works by the authors for various operators: elastostatics, stationary conduction problems and flow in saturated porous media. Several new applications are presented. The first part of the paper is devoted to the 
presentation of the data completion problem, and then the energy-like error method is developed. Then, the minimization algorithm used which is a sensitive point when dealing with ill-posed problems, is detailed. The last part is devoted to various applications, where the performance and robustness of the method are examined in different situations. The general energy-like error approach developed here can be linked with pioneering works, such as those of Knowles who identified coefficient functions in an elliptical equation [11] and [12] in a similar framework, and more generally with the development of constitutive equation errors for various applications by Ladevèze et al. in $[13,14,15]$.

\section{DATA COMPLETION PROBLEM FOR LINEAR SYMMETRIC OPERATORS}

Let us consider a positive symmetric bilinear form $a$ and a space $V$ of functions in a domain $\Omega$, such that $a(u, v)$ is finite for any pair of fields $(u, v)$ of $V^{2}$. Then, $B$ represents the associated natural boundary condition operator, defined by:

$$
\forall u \in V: \quad \int_{\partial \Omega} \text { Bu.nv }=a(u, v), \quad \forall v \in V
$$

where $\boldsymbol{n}$ stands for the external unit vector to the regular boundary $\partial \Omega$ of the domain. Now let us suppose that this external boundary is partitioned into three non overlapping parts, $\partial \Omega=\Gamma_{m} \cup \Gamma_{b} \cup \Gamma_{u}, \Gamma_{m} \cap \Gamma_{b}=\Gamma_{m} \cap \Gamma_{u}=\Gamma_{u} \cap \Gamma_{b}=\emptyset$ :

- On the boundary $\Gamma_{m}$, natural boundary conditions $B u . n=T_{m}$ and an essential boundary conditions $u=U_{m}$ are known or measured,

- On the boundary $\Gamma_{b}$, a usual combination of natural boundary conditions $(B u \cdot \boldsymbol{n})_{i}=b_{i}$ and essential boundary conditions $u_{j}=U_{j}$ is known. We introduce the linear form $l$ and the following subspaces:

$$
\left.l(v)=\int_{\Gamma_{b}} b_{i} v_{i}, \quad V_{U}=\left\{v \in V,\left.v_{j}\right|_{\Gamma_{b}}=U_{j}\right\} \quad \text { and } \quad V_{0}=\left\{v \in V,\left.v_{j}\right|_{\Gamma_{b}}=0\right\}\right)
$$

- On the boundary $\Gamma_{u}$, both natural and essential boundary conditions are unknown.

The data completion problem is therefore the following:

Find a field $\mathrm{u}$ in space $V_{U}$ such that:

$$
\left\{\begin{array}{l}
a(u, v)=\int_{\Gamma_{u}} \text { Bu.nv }+\int_{\Gamma_{m}} T_{m} v+l(v), \quad \forall v \in V_{0} \\
\left.u\right|_{\Gamma_{m}}=U_{m}
\end{array}\right.
$$

That means: finding an equilibrium field meeting the usual boundary conditions on $\Gamma_{b}$ and the superabundant boundary conditions on $\Gamma_{m}$. If such a field exists and is unique, it provides the lacking boundary conditions $(B u . \boldsymbol{n}, u)$ on $\Gamma_{u}$. This feature justifies considering (3) as a data completion problem. If part $\Gamma_{b}$ of the boundary vanishes, the problem turns out to be a Cauchy problem. In the following table, the corresponding spaces, bi-linear forms and boundary operators are displayed for the stationary thermal conduction problem-or incompressible Darcy equations for saturated porous media, and for the elastostatic problem. $\mathbf{k}$ is the thermal conductivity tensor or the ratio of the permeability tensor over dynamic viscosity and $\mathbf{A}$ the Hooke tensor. In the data completion problem, they are assumed to be known over the whole domain $\Omega$. When $u$ is a vector field, as in elastostatics, more elaborated data completion problems can be defined by using 
Table 1. Variational formulations

\begin{tabular}{|l|c|c|}
\hline V Space & a form & B operator \\
\hline$H^{1}(\Omega)$ & $a(u, v)=\int_{\Omega} k: \nabla u . \nabla v$ & $B u . n=k: \nabla u . n$ \\
\hline$H^{1}(\Omega)^{3}$ & $a(u, v)=\int_{\Omega} A: \varepsilon(u): \varepsilon(v)$ & $B u . n=A: \varepsilon(u) . n$ \\
\hline
\end{tabular}

alternative combinations of superabundant data on $\Gamma_{m}$. For example, in data imaging devices, only tangential displacement fields on a stress free surface are measured, thus the question of expanding the displacement field inside the solid can be called an incomplete Cauchy problem. Although the superabundant data are $B u . n=T_{m}$ and $u-(u . n) n=U_{m}^{t}$, where $U_{m}^{t}$ is the tangential component of $U_{m}$, the method developed here can be applied without any major change. This has been addressed by Andrieux and Baranger in [16], where 3D applications are also given.

\section{FORMULATION OF THE DATA COMPLETION PROBLEM AS AN ENERGY ERROR MINIMIZATION PROBLEM}

The data completion problem is not a classical one because of the combination of superabundant data in a part of the boundary and a total lack of data in another part. To derive a general solution method, we proceed in two steps. First two well-posed usual direct problems are defined, the solutions of which are denoted by $u_{1}$ and $u_{2}$. These problems are parameterized respectively by two fields $\mathrm{g} \tau$ (a natural boundary condition quantity) and $v g$ (a field quantity or Dirichlet boundary condition) defined on the boundary $\Gamma_{u}$ where no data are available, namely:

$$
\begin{gathered}
u_{1} \in V_{U}, \quad\left\{\begin{array}{l}
a\left(u_{1}, v\right)=\int_{\Gamma_{u}} \tau v+l(v), \quad \forall v \in V_{0}^{m} \\
\left.u_{1}\right|_{\Gamma_{m}}=U_{m}
\end{array}, \quad V_{0}^{m}=\left\{v \in V_{0},\left.v\right|_{\Gamma_{m}}=0\right\}\right. \\
u_{2} \in V_{U}, \quad\left\{\begin{array}{l}
a\left(u_{2}, v\right)=\int_{\Gamma_{m}} T_{m} v+l(v), \quad \forall v \in V_{0}^{u} \\
\left.u_{2}\right|_{\Gamma_{u}}=v
\end{array}, \quad V_{0}^{u}=\left\{v \in V_{0},\left.v\right|_{\Gamma_{u}}=0\right\}\right.
\end{gathered}
$$

The first problem has a prescribed field condition with the actual value $U_{m}$ on $\Gamma_{m}$, and a natural boundary condition with value $\tau$ on $\Gamma_{u}$, whereas the second corresponds to prescribed value $v$ on $\Gamma_{u}$ and a natural boundary condition corresponding to the actual value $T_{m}$ on $\Gamma_{m}$. For the second step, it should now be noted that if the two fields $u_{1}(\tau)$ and $u_{2}(v)$ coincide, then the data completion problem is solved: the common value $u=u_{1}=u_{2}$ indeed satisfies (3) and:

$$
\left.u\right|_{\Gamma_{u}}=v \quad,\left.\quad B u \cdot n\right|_{\Gamma_{u}}=\tau
$$

It is then natural to introduce a functional measuring the gap between the two fields. Here, the choice of this functional is the error in the energy semi-norm:

$$
J\left(u_{1}, u_{2}\right)=\frac{1}{2} a\left(u_{1}-u_{2}, u_{1}-u_{2}\right) .
$$

This denomination of energy is chosen because of the minimum properties (minimum energy theorems) characterizing the two solutions of problems (4) and (5):

$$
u_{1}=\begin{aligned}
& \operatorname{ArgMin} \\
& \left\{v \in V_{0}^{m},\left.v\right|_{\Gamma_{m}}=U_{m}\right\}
\end{aligned} \quad \frac{1}{2} a(v, v)-\int_{\Gamma_{u}} \tau v-l(v)
$$




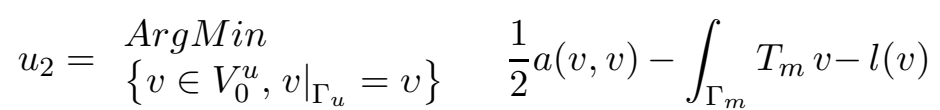

The data completion problem is then formulated via the minimization of the energy error functional (7):

$$
\begin{aligned}
& \text { Find }(\tau, v) \text { that minimize } E(\tau, v) \equiv J\left(u_{1}, u_{2}\right) \\
& \text { With } \begin{cases}u_{1}=u_{1}\left(U_{m}, \tau\right) & \text { solution of }(4) \\
u_{2}=u_{2}\left(T_{m}, v\right) & \text { solution of }(5)\end{cases}
\end{aligned}
$$

Obviously $E$ is a positive quadratic and convex functional because $a(\bullet, \bullet)$ is a positive bilinear form. It is also straightforward to verify that if a pair of fields $(\tau, v)$ satisfies $E=0$ then the corresponding fields $u_{1}$ and $u_{2}$ possibly differ only by a field belonging to the null space of the bilinear form $a(\bullet, \bullet)$ (if it is not reduced to zero) satisfying null conditions on $\Gamma_{b}$, and that $u_{1}$ solves the Cauchy problem.

$$
E(\tau, v)=J\left(u_{1}, u_{2}\right)=0 \Leftrightarrow,\left\{\begin{array}{c}
u_{1} \text { solves }(2) \text { and } u_{2}=u_{1}+r \\
a(r, v)=0 \forall v \in V,\left.r_{j}\right|_{\Gamma_{b}}=0
\end{array}\right.
$$

For computational purposes, an alternative form of the functional can be derived, involving only boundary integrals, by using the weak formulation of problems (4) and (5):

$$
E(\tau, v)=\frac{1}{2} \int_{\Gamma_{m}}\left(B u_{1}(\tau) \cdot n-T_{m}\right) \cdot\left(U_{m}-u_{2}(v)\right)+\frac{1}{2} \int_{\Gamma_{u}}\left(\tau-B u_{2}(v) \cdot n\right) \cdot\left(u_{1}(\tau)-v\right)
$$

This expression is used in the computations and avoids any domain integration. Note that it involves both surfaces $\Gamma_{m}$ and $\Gamma_{u}$.

\section{TRUST REGION ALGORITHM AND GRADIENT COMPUTATION FOR THE EFFECTIVE MINIMIZATION OF THE ENERGY ERROR}

The data completion problem has been reduced to the minimization of a quadratic, convex, functional (10) and at first glance, the best way of achieving minimization is to solve the first order optimality condition:

$$
\nabla E(\tau, v) \cdot(\delta \tau, \delta v)=0 \quad \forall(\delta \tau, \delta v)
$$

which turns out to be a linear problem. Once discretized, equation (13) is therefore a linear system where the unknowns are the components of the vector collecting the numerical values describing the discretized form of field $v$ gnd natural boundary quantity $\tau$ on boundary $\Gamma_{u}$. However, as soon as the number of unknowns increases, as is the case for $3 \mathrm{D}$ data completion problems for vector fields, this method has to be discarded for two main reasons. First the computational costs of the Hessian matrix of the discretized error energy $E$ increases dramatically as it requires the resolution of $n$ problems of type (4) or (5), $n$ being the total number of unknowns. Secondly, the data completion problem or the equivalent Cauchy problem is known to be ill-posed. In fact, even if there only one solution exists when the data are compatible, there is no continuity of the solution with respect to the data pair $\left(U_{m}, T_{m}\right)$. This feature results in very poor conditioning of the Hessian matrix of $E$, the condition number decreasing very quickly as the number of unknowns increases, Andrieux and Baranger $[15,16,17]$. The direct resolution of the linear 
system resulting from (13) leads to very unstable results and is very sensitive to the noise inevitably present in the data $\left(U_{m}, T_{m}\right)$.

A direct optimization method is preferred, using only the gradients of the function. The gradient must be computed by an adjoint method because of the implicit dependence of the $u_{1}$ and $u_{2}$ fields on variables $(\tau, v)$, and the relatively high cost of evaluation of the function itself. The Lagrangian associated with the energy error function (7) with the state equations (4) and (5) is:

$$
\begin{aligned}
& L\left(u_{1}, u_{2}, \mu, \lambda, v_{1}, v_{2} ; \tau, v\right)=J\left(u_{1}, u_{2}\right)+a\left(u_{1}, v_{1}\right)+a\left(u_{2}, v_{2}\right)-l\left(v_{1}+v_{2}\right) \\
& -\int_{\Gamma_{m}} T_{m} \cdot v_{2}-\int_{\Gamma_{u}}\left[\lambda .\left(u_{2}-v\right)+\mu . v_{2}+\tau . v_{1}\right]
\end{aligned}
$$

and is defined on the following product space:

$$
\left(u_{1}, u_{2}, v_{1}, v_{2}, \lambda, \mu\right) \in V_{U}^{m} x V_{U} x V_{0} x V_{0}^{u} x H\left(\Gamma_{u}\right) x H\left(\Gamma_{u}\right)
$$

With $V_{U}^{m}=\left\{v \in V_{U},\left.v\right|_{\Gamma_{m}}=U_{m}\right\}, H\left(\Gamma_{u}\right)$ is the traces' dual space on $\Gamma_{u}$ of the fields belonging to $V$. Following the classical approach, the stationarity of the Lagrangian with respect to primal fields $u_{1}$ and $u_{2}$ leads to the definition of the two adjoint problems:

$$
\begin{aligned}
& v_{1} \in V_{0}^{m}, \quad a\left(v_{1}, v\right)=a\left(u_{1}-u_{2}, v\right) \forall v \in V_{0}^{m} \\
& v_{2} \in V_{0}^{u}, \quad a\left(v_{2}, v\right)=a\left(u_{2}-u_{1}, v\right) \forall v \in V_{0}^{u}
\end{aligned}
$$

These adjoint problems are quite simple, because of the symmetry of the bilinear form $a$, which leads to the self-adjoint property for the associated operator. They are also linear problems, and because of the energy choice for the semi-norm used in the quantification of the gap between $u_{1}$ and $u_{2}$, only boundary conditions involving the data and the primal fields appear. Furthermore the operator, or rigidity matrix in the discretized version, is the same as for the direct problems (4) and (5). With these adjoint fields, the gradients of the error functional are simply:

$$
\nabla_{\tau} E(\tau, v)=-\left.v_{1}\right|_{\Gamma_{u}}, \quad \nabla_{v} E(\tau, v)=\left.\left[B u_{2} . n+B v_{2} . n-\tau\right]\right|_{\Gamma_{u}}
$$

The derivation of the discrete adjoint problems, which must be preferred to the discretized version of the continuous adjoint problem (16), follows exactly the same path.

Going back to the minimization algorithm for the discretized $E$ function, each iteration of the algorithm involves the solution of four linear systems: two direct problems defined in (4) and (5) and two adjoint problems defined by (16). The quadratic energy error function can be written formally as follows:

$$
\begin{aligned}
& E(X)=\frac{1}{2} X^{T} H X+X^{T} B+C \\
& \text { with } \quad X=\left\{X_{\tau}, X_{v}\right\} \text { gathering the unkowns for } \tau \text { and } v \text { respectively }
\end{aligned}
$$

where $H$ is the Hessian which depends only on the geometrical and material data, while $B$ and $C$ depend on the geometrical and material data and also on the measured boundary data $\left(T_{m}, U_{m}\right)$. The Hessian is positive and undefined, so the convergence of the conjugate gradient algorithm is slow. The Trust Region Method (TRM) is adopted in order to optimize the computational cost. The latter method must solve the following sub-problem 
for each iteration $k$ :

$$
\min \left\{Q\left(S_{k}\right)=\frac{1}{2} S_{k}^{T} H S_{k}+S_{k}^{T} \nabla E_{k-1} \text { such that }\left\|S_{k}^{T}\right\| \leq \delta\right\}
$$

where $Q\left(S_{k}\right)$ is the quadratic approximation of $E\left(X_{k-1}+S_{k}\right), \nabla E_{k-1}=H X_{k-1}+B$ is the energy function gradient computed at the previous iteration $(k-1)$, and $\delta$ is a positive scalar which measures the radius of the trust region and reflects the confidence in the second-order model. As the energy function $E$ is quadratic, $Q\left(S_{k}\right)$ is an exact approximation of $E\left(X_{k-1}+S_{k}\right)$, while the optimal search direction is Newtonian: $S_{k}=-H^{-1} \nabla E_{k-1}$. However, this search direction is not well defined when the Hessian is singular and the gradient depends on the superabundant data. The search direction is instead defined by solving the Trust Region Sub-problem defined by (19) whose associated Lagrangian function is:

$$
L(X, \lambda)=\frac{1}{2} S_{k}^{T} H S_{k}+S_{k}^{T} \Delta E_{k-1}+\frac{\lambda}{2} S_{k}^{T} S_{k}
$$

This quadratic function can be interpreted as a regularized quadratic model for $E$ around $X_{k-1}$, and its exact solution is given by:

$$
(H+\lambda I) S_{k}=-\nabla E_{k-1}
$$

The proposed approach using the minimization formulation (18) does not explicitly incorporate any kind of regularization, although the Cauchy problem is very ill-posed. Nevertheless, the use of the trust region method brings a form of regularization to the numerical applications and the value of the stopping criterion plays the role of regularization parameter. For moderate noise corrupting the data, this regularization appears as sufficient as shown for the thermal conductivity equation (Andrieux et al. [17]). The stopping criterion used in the optimization process of the following applications is the function value or its relative change between two iterations.

\section{APPLICATIONS}

In this section, we present different applications where the energy method is used to identify the boundary conditions. These applications concern thermostatics and elastostatics.

\subsection{Thermostatics}

We consider a domain $\Omega$ as shown in figure 2, where the temperature and flux are known on $\Gamma_{m}$. The goal is to identify the temperature and the flux on $\Gamma_{u}$, which has a geometric singularity at point $O$. We consider the following temperature distribution: $u=$ $y /\left((x-0.5)^{2}+y^{2}\right)$. Notice that this field has very large temperature variations and a flux discontinuity at the point $O$ on $\Gamma_{u}$. The superabundant data are then generated by using the above analytical distribution as the Dirichlet boundary condition of the direct problem. Figures 3 and 4 show the identified temperature and flux obtained by finite element analysis with a mesh characterized by 73 nodes on $\Gamma_{m}$ and 37 nodes on $\Gamma_{u}$, see figure 2. Notice that the identified fields are in good agreement with the exact ones, and that the flux discontinuity is recovered with good precision. The stopping criterion for the 
Trust Region Method is set at $10^{-12}$ and the minimum of the functional is reached after 44 iterations.

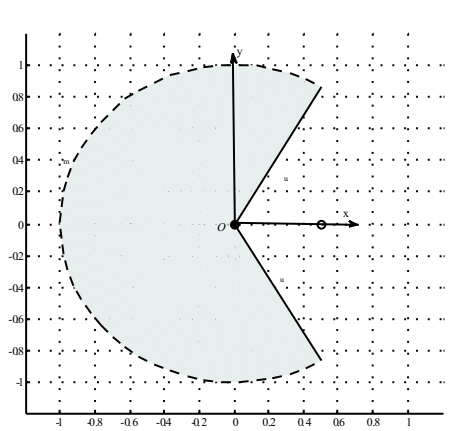

Fig. 1. Geometric data

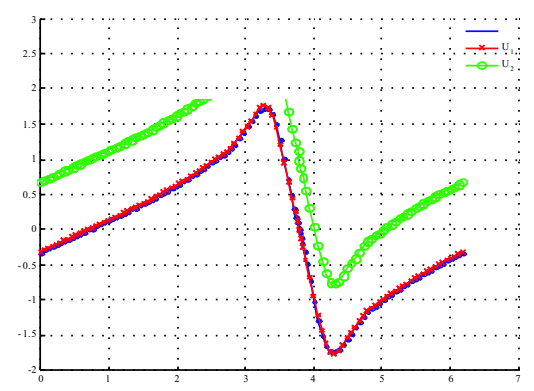

Fig. 3. $U_{e}$ is the exact temperature on $\Gamma_{u}$. $\mathrm{U}_{1}$ and $\mathrm{U}_{2}$ are the identified temperatures

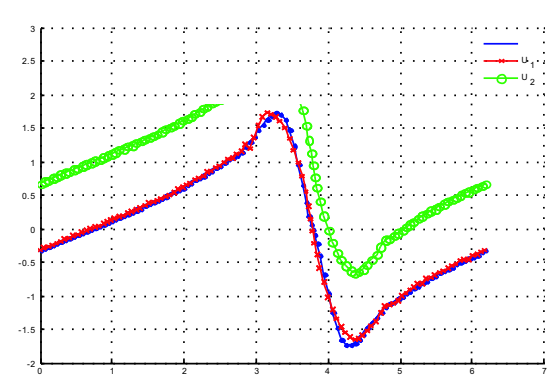

Fig. 5. $\mathrm{U}_{e}$ is the exact temperature on $\Gamma_{u}$. $\mathrm{U}_{1}$ and $\mathrm{U}_{2}$ are the identified temperatures with noisy data $(5 \%)$

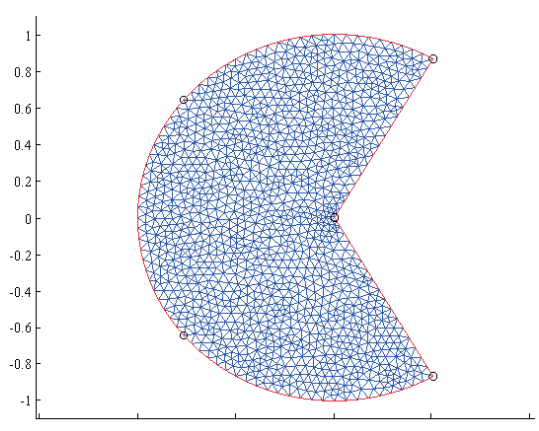

Fig. 2. Mesh of the domain $\Omega$

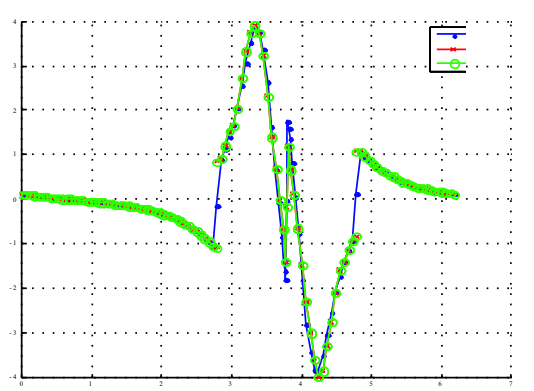

Fig. 4. $\nabla \mathrm{U}_{e} \cdot \mathrm{n}$ is the exact flux on $\Gamma_{u} \cdot \nabla \mathrm{U}_{1} \cdot \mathrm{n}$ and $\nabla \mathrm{U}_{2} \cdot \mathrm{n}$ are the identified fluxes

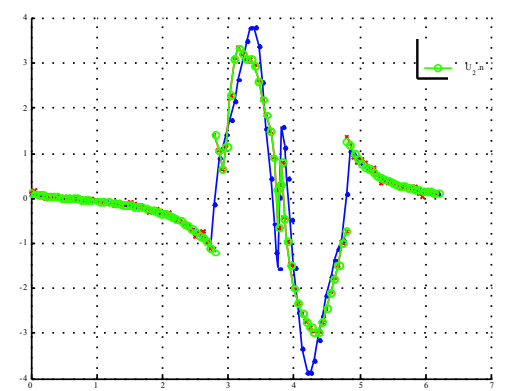

Fig. 6. $\nabla \mathrm{U}_{e} \cdot \mathrm{n}$ is the exact flux on $\Gamma_{u} . \quad \nabla \mathrm{U}_{1} . \mathrm{n}$ and $\nabla \mathrm{U}_{2} . \mathrm{n}$ are the identified fluxes with noisy data $(5 \%)$ 
Figs. 5 and 6 show the identified temperature and flux obtained with noisy data $(5 \%)$ on $\Gamma_{m}$. These results are obtained without additional regularization. The stopping criterion for the Trust Region Method is set at $10^{-3}$, and convergence is reached after only three iterations.

In this paragraph we present a problem governed by a Laplace operator for homogenous media with geometric and field singularities. The corresponding data completion problem is solved with good accuracy, even for reasonably noisy data (5\%). This method has also been applied for strongly orthotropic media and has also given good results, see Andrieux et. al. [17]. Two other applications have also yielded very good results, the first application being devoted to the identification of leaks in porous media; see Escriva and Baranger [18]. The second is devoted to electrocardiography, where the heart's potential is identified by using the measured potential in the thorax; see Hariga and Baranger [19].

\subsection{Elastostatics}

\section{A. Identification of elastic moduli for a geometrically determined inclusion}

Here, we consider a body $\Omega=\Omega_{1} \cup \Omega_{2}$ with a geometrically determined inclusion $\Omega_{2}$, as shown in Fig. 7. The material parameters (Young's modulus and Poisson's ratio) of the inclusion are unknown. We propose a method to identify these parameters by using superabundant boundary conditions measured on a part of the outer boundary. Two steps are necessary: the first consists of solving the corresponding Cauchy problem defined on the domain $\Omega_{1}$ alone. This leads to identifying boundary conditions $(\tau, v)$ on the inner boundary, as shown on figure 8 . The second step consists in applying the Maxwell-Betti reciprocity theorem by considering inclusion $\Omega_{2}$ alone and using the identified data $(\tau, v)$. We consider domain $\Omega_{1}$, as shown on figure 8 , where the displacements and the surface tractions on the lateral outer boundaries are known, the horizontal outer boundaries are free from surface tractions and the data $(\tau, v)$ on the inner boundary are unknown. The superabundant data are generated numerically by solving the direct problem defined on the figure 7. By solving the minimization problem defined in (11) the lacking boundary conditions are identified. The minimization process is achieved with 6 iterations. Figures 9 and 10 show the identified and the exact data. Notice that the results obtained are in good agreement with the exact ones.

The Cauchy problem considered addresses a homogeneous isotropic two-dimensional medium under a plane strain assumption, i.e. with reference to table 1:

$$
a(u, v)=\int_{\Omega} A: \varepsilon(u): \varepsilon(v) \text { with } A=(k-\mu) I_{2} \otimes I_{2}+2 \mu I_{4}
$$

where $k$ is the compressibility modulus and $\mu$ the shear modulus. To identify the material properties $(k, \mu)$ of the inclusion, we use the pair of the boundary conditions identified above denoted here by $\mathrm{U}=\left\{\mathrm{U}_{x}, \mathrm{U}_{y}\right\}^{T}$ and $\mathrm{F}=\left\{\mathrm{F}_{x}, \mathrm{~F}_{y}\right\}^{T}=\sigma . \boldsymbol{n}$ and we consider the domain $\Omega_{2}$ of the inclusion alone. An auxiliary displacement field $w$ is then chosen such that it satisfies the elastic equilibrium equation. Applying the Betti-Maxwell reciprocity equation, we can determine a set of equations on $\mathbf{A}$, by choosing various expressions of the auxiliary field $w$ :

$$
A: \int_{\partial \Omega_{2}} \varepsilon(w): n . U=\int_{\partial \Omega_{2}} F . w
$$




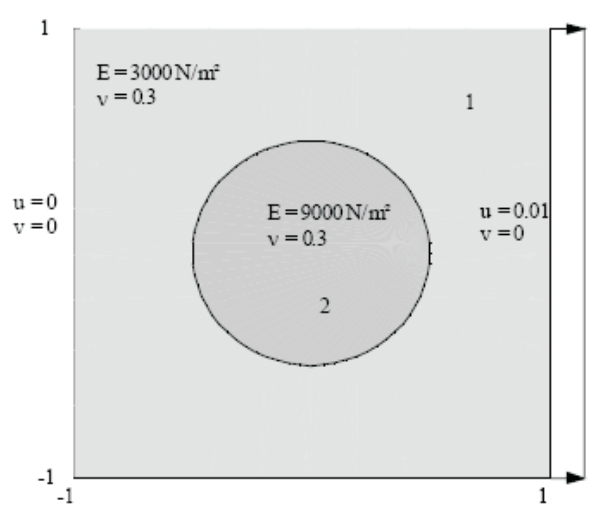

Fig. 7. Geometry and materials data of the direct problem

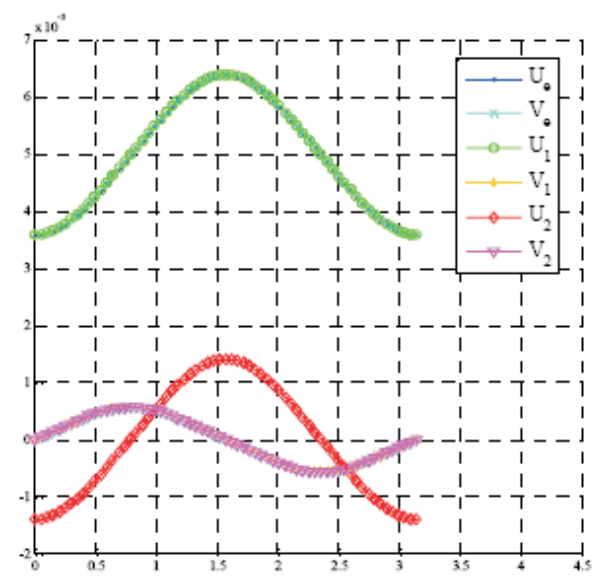

Fig. 9. Exact and identified displacements components on $\Gamma_{u}$

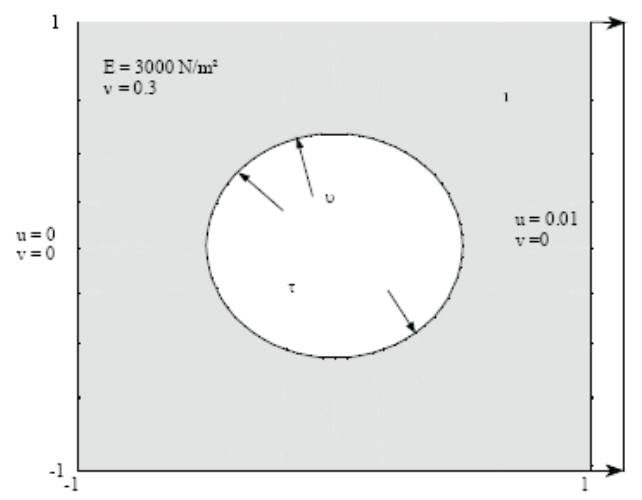

Fig. 8. Geometry and material data used to solve the Cauchy problem

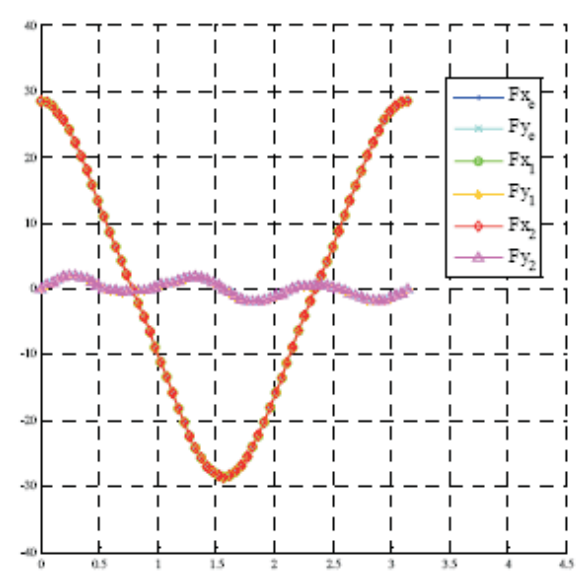

Fig. 10. Exact and identified stress vector component on $\Gamma_{u}: F=\left\{F_{x}, F_{y}\right\}^{T}=\sigma . n, n$ is the outer normal at $\Gamma_{u}$

Taking $w=x e_{x}+y e_{y}$ and if $\int_{\partial \Omega_{2}} U . n d \partial \Omega_{2}$ does not vanish, which is the case here, compressibility modulus $k$ is determined as follows:

$$
k=\frac{\int_{\partial \Omega_{2}} F_{x} x+F_{y} y}{2 \int_{\partial \Omega_{2}} U_{x} n_{x}+U_{y} n_{y}}
$$

In order to identify the shear modulus another auxiliary field $w=(y-x) e_{x}+(x+$ $y) e_{y}$ is chosen. This choice leads to the following formula:

$$
\mu=\frac{1}{2} \frac{\int_{\partial \Omega_{2}} F_{x}(y-x)+F_{y}(x+y)}{\int_{\partial \Omega_{2}} U_{x}\left(n_{y}-n_{x}\right)+U_{y}\left(n_{x}+n_{y}\right)}
$$


Table 2 shows the identified parameters compared to the exact ones. It should be noted that the precision is very good, as the relative error does not exceed $2 \%$ for $E$ and $\mu$.

Table 2. Parameter formulae with the plane strain assumption. Identified and exact parameter values

\begin{tabular}{|l|c|c|c|c|}
\hline Parameters & $k$ & $\mu$ & $E$ & $\nu$ \\
\hline Exact & 8653.8 & 3461.5 & 9000 & 0.3 \\
\hline Identified & $8311.5(3.9 \%)$ & $3432.7(0.8 \%)$ & $8880.4(1.3 \%)$ & $0.2935(2.1 \%)$ \\
\hline $\begin{array}{l}\text { Plane strain } \\
\text { formulas }\end{array}$ & $k=\lambda+\mu \quad \lambda=\frac{E \nu}{(1+\nu)(1-2 \nu)}$ & $\mu=\frac{E}{2(1+\nu)} \quad E=\frac{\mu}{k}(2 k-\mu) \quad \nu=\frac{k-\mu}{2 k}$ \\
\hline
\end{tabular}

\section{B. Recovery of stress intensity factors of a cracked body}

This example concerns a plate with a crack. The goal here is to identify the stress intensity factor. The geometric line where the crack is located is assumed to be known and only the half of the plate is modelled due to its symmetry, see figure 20. The superabundant data are generated numerically by solving the direct problem. We consider two models for the Cauchy problem:

-c Model 1: we consider domain $\Omega=\Omega_{1} \cup \Omega_{2} \cup \Omega_{3}$ with superabundant data on $\Gamma_{m}$ and unknown one on $\Gamma_{u 1}$.

- Model 2: we consider domain $\Omega=\Omega_{1} \cup \Omega_{2}$ with superabundant data on $\Gamma_{m}$ and unknown data on $\Gamma_{u 2}$.

Figs. 12 and 13 show the exact and identified displacements fields obtained with the model 1 and 2, respectively. Note the good agreement between the two fields. Table 3 presents the stress intensity factors of mode I, evaluated by using the direct problem and the two inverse problems based on models 1 and 2. The stress intensity factor is evaluated using the so-called $J$-integral. For the mode I, the $J$-integral is related to the stress intensity factor and the elastic material,for the plane stress case, as follows:

$$
K_{I}=\sqrt{E J} \text { with } J=\int_{\Gamma_{i}} W n_{x}-\sigma_{i j} n_{j} u_{i, x} d S
$$

where $E$ is the Young modulus.

The problem addressed here has an analytical solution for the stress intensity factor: $K_{I}=\sigma \sqrt{\pi a} f$, where $\sigma=20 \mathrm{MPa}$ is the tensile load, $a=0.6$ is the crack length and $f=2.1$ is the configuration correction factor. This factor is determined for this configuration using a polynomial equation, see Ragab et. al. [20].

Table 3 shows the stress intensity factors computed by the direct method and the inverse problem based on the models defined above. The first line shows the stress intensity factor obtained by the $J$-integral on boundaries 1,2 and 3 . The second line shows the stress intensity factors obtained by solving the Cauchy problem on model 1 . Here the data completion is held on the boundary $\Gamma_{u 1}$ and the $J$-integrals are evaluated further on boundaries1, 2 and 3. The results are in good agreement with those obtained analytically. The third line shows the stress intensity factors obtained by solving the Cauchy problem 


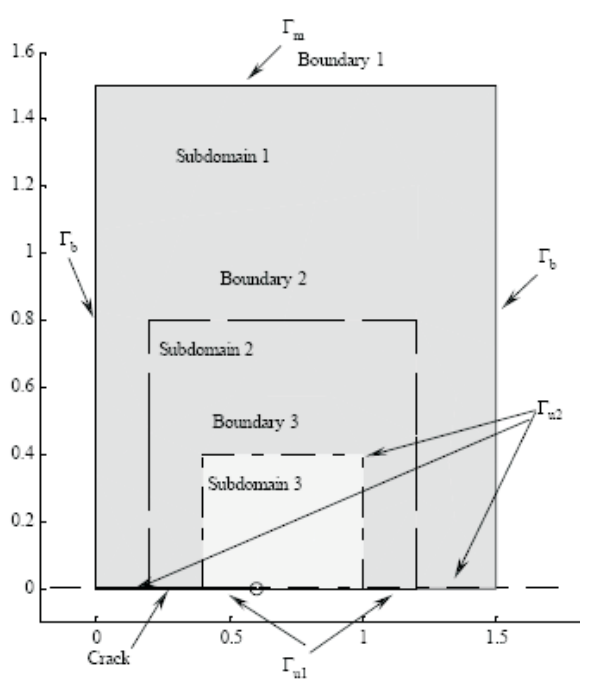

Fig. 11. Geometric data

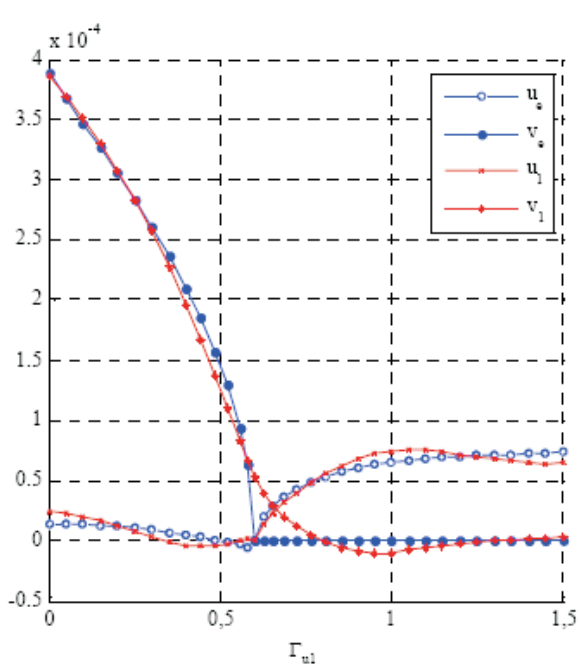

Fig. 12. Exact and identified displacement components on $\Gamma_{u 1}$

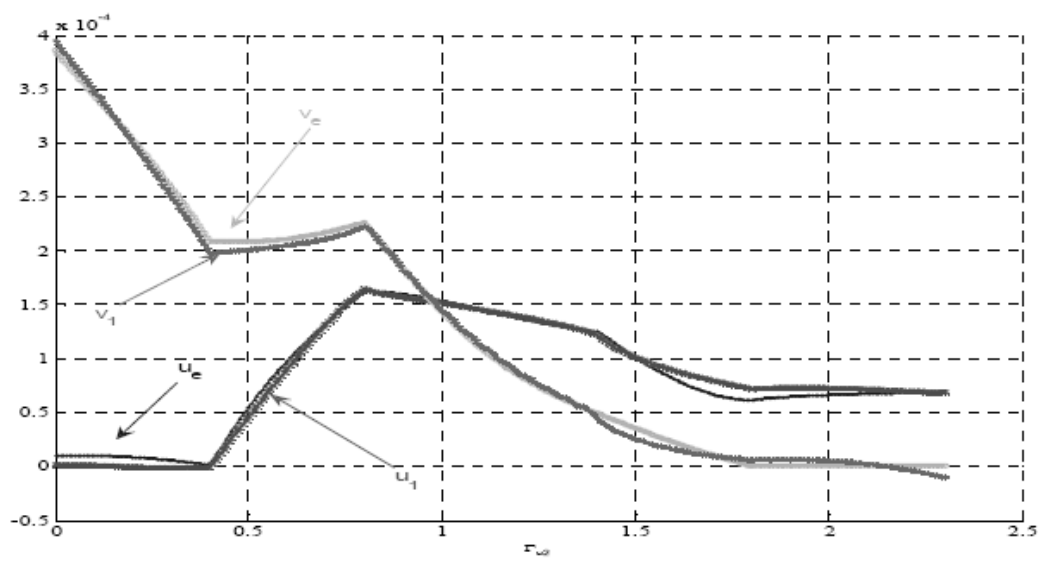

Fig. 13. Exact and identified displacement components on $\Gamma_{u 2}$

on model 2. Here, the data completion is held on boundary $\Gamma_{u 2}$ and the $J$-integrals are evaluated further on boundaries 1,2 and 3. It should be noted that the relative error is higher for the stress intensity factor obtained from the $J$-integral evaluated on boundary 1 , which is a part of boundary $\Gamma_{u 2}$, and also on boundary 3 . This can be explained by the fact that the $J$-integral is evaluated on a boundary whose data are identified and show greater disturbance, as shown in figure 12.

\section{Expansion of surface displacement fields inside an elastic solid}

The last example concerns the expansion of surface displacement fields inside an elastic domain. We consider the domain shown in figure 14, which can be semi-infinite and where loads denoted by $p$ act on a part of the free surface. It is assumed that loads $p$ and the boundary where they are acting are unknown. the domain $\Omega$ defined by the zoom 
Table 3. Comparison of the stress intensity factors obtained from the direct and inverse problem. The reference solution is the analytical one given by $K=57.66$ $\mathrm{MPa} \mathrm{m} \mathrm{m}^{1 / 2}$

\begin{tabular}{|l|c|l|c|}
\hline $\begin{array}{l}K_{i} \text { evaluated on } \\
\text { the boundary } i\end{array}$ & $K_{1}\left(\mathrm{MPa} \mathrm{m}^{1 / 2}\right)$ & $K_{2}\left(\mathrm{MPa} \mathrm{m}^{1 / 2}\right)$ & $K_{3}\left(\mathrm{MPa} \mathrm{m}^{1 / 2}\right)$ \\
\hline Direct Model & $57.69(0.4 \%)$ & $57.68(0.03 \%)$ & $57.66(0.05 \%)$ \\
\hline $\begin{array}{l}\text { Inverse problem: } \\
\text { Model 1: } \Omega=\Omega_{1} \cup \Omega_{2} \cup \Omega_{3}\end{array}$ & $58.11(0.7 \%)$ & $58.77(1.9 \%)$ & $58.17(0.8 \%)$ \\
\hline $\begin{array}{l}\text { Inverse problem: } \\
\text { Model 2: } \Omega=\Omega_{1} \cup \Omega_{2}\end{array}$ & $54.88(4.8 \%)$ & $57.84(0.3 \%)$ & $55.87(3.1 \%)$ \\
\hline
\end{tabular}

is considered, as shown in figure 15. The displacement measurement can be performed on the free surface of $\Omega$ denoted here by $\Gamma_{m}$. Thus superabundant boundary conditions are available on $\Gamma_{m}$, i.e. Dirichlet boundary conditions (measurements) and Neumann boundary conditions (free traction surface). Note here that the Neumann boundary condition is known exactly, whereas the boundary conditions are unknown on the rest of the boundary denoted by $\Gamma_{u}$, such that $\partial \Omega=\Gamma_{m} \cup \Gamma_{u}$.

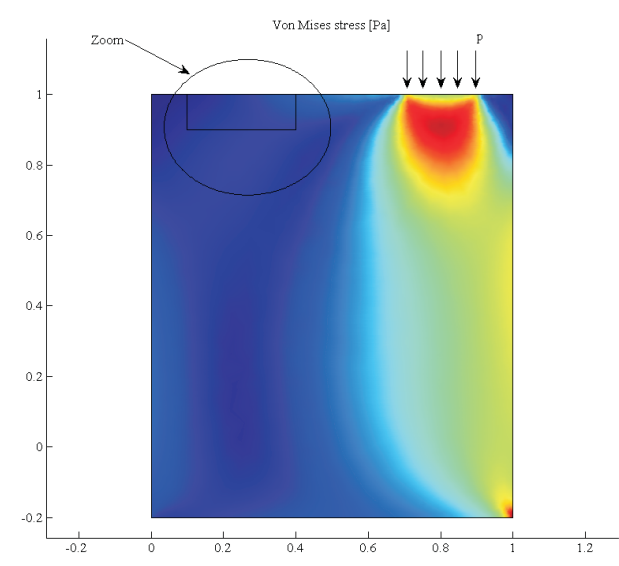

Fig. 14. Forward problem data

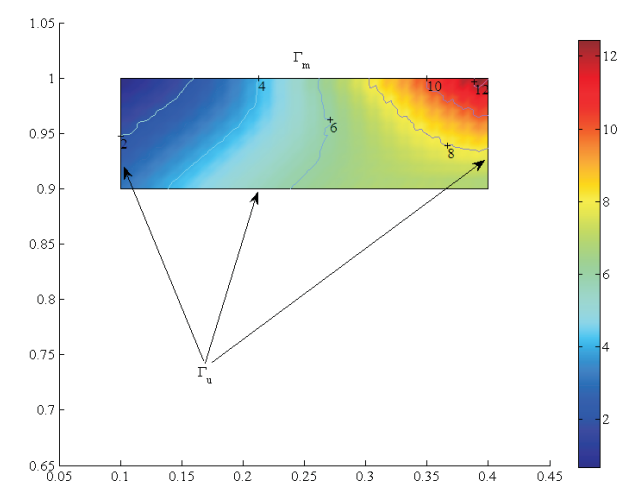

Fig. 15. Exact Von Mises stress distribution on the domain defined by the zoom (figure 14)

To address this problem we first consider a plane strain finite element model and an elastic material with Young's modulus $E=1000 \mathrm{MPa}$ and Poisson ratio $\nu=0.3$. Then the direct problem is solved under the action of load $p=100 \mathrm{~N} / \mathrm{m}$. Figures 14 and 15 show the map of the Von Mises stress distribution. The displacement measurements on $\Gamma_{m}$ to be used in the inverse problem are extracted from this direct problem.

Figures 16 and 17 show the identified displacements components and Von Mises stress distribution. It can be seen that the identified data are in very good agreement with the exact ones. 


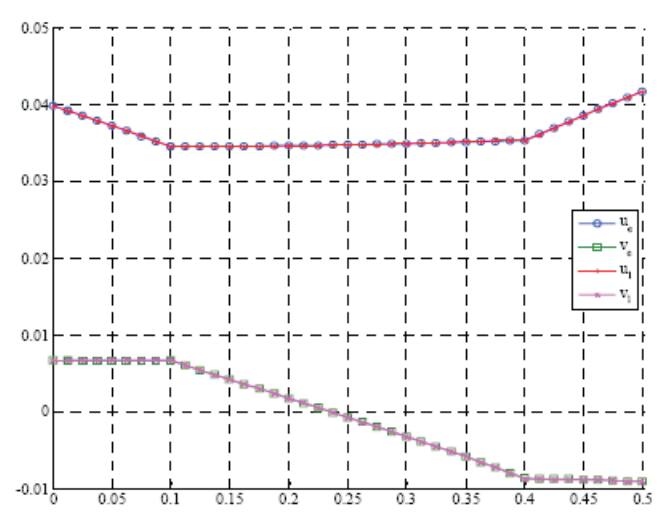

Fig. 16. Exact and Identified displacement vector components on $\Gamma_{u}$

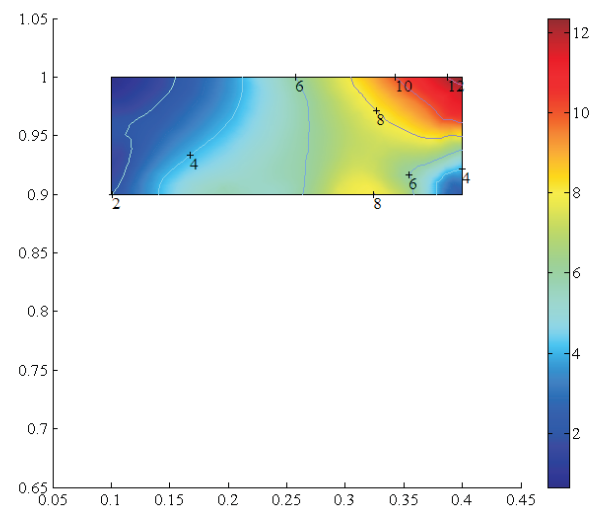

Fig. 17. Identified Von Mises stress distribution on the subdomain defined by the zoom (figure 14)

This example has two main difficulties. First, boundary $\Gamma_{m}$ is much smaller than $\Gamma_{u}$, thus there are fewer data than unknowns. Second, surface traction $T_{m}$ on $\Gamma_{m}$ is zero; consequently the problem defined by (5) is ill-posed in the sense that the Dirichlet boundary condition $v$ must be initialized with an adequate value in order to avoid rigid body movements.

\section{CONCLUSION}

A general method has been presented in this paper to solve Cauchy problems for symmetric elliptic operators such as those found in elastostatics and thermostatics and thus identify boundary conditions. Four examples have been presented. The first deals with thermostatics and in spite of a high temperature variation in the vicinity of the source point and the discontinuity of the flux, the missing data are recovered with good precision even when the measured data are noisy (5\%). This example allows the generalization of the method to a physical problem governed by a Laplace operator. The three other examples deal with elastostatics problems.

The results obtained are in good agreement with the exact ones, even when the context and conditions are unfavourable: the quantity of known data on the boundary $\Gamma_{m}$ is significantly less than that of unknown data on $\Gamma_{u}$; the boundary $\Gamma_{u}$ exhibits geometric singularities, such as cracks. Obviously, it is essential that the user of this method must have thorough knowledge of the physical problem so that they can better interpret the results and, in particular, better control the main stages: initializing the variables to be identified and controlling minimization with an adequate stopping criterion.

The method presented can be easily implemented in industrial finite element software in order to solve industrial identification problems. No regularization is needed. However, in the case of industrial applications, the data are often severely altered by noise which also alters identification. The effect of noise has been examined for the Cauchy problem for the Laplacian operator in a previous paper $[16,17,18]$, dealing with thermostatic 
and elastostatic examples. The incorporation of some kind of regularization seems to be a requisite in the method as soon as noise exceeds $5 \%$.

This method is being extended and investigated for nonlinear problems (material and geometric nonlinearities) and for time dependent problems [23,24,25].

\section{REFERENCES}

[1] Sutton M.A., Cheng M., Peters W.H., Chao Y.S., McNeill S.R., Application of an optimized digital correlation method to planar deformation analysis, Image vision Computing 4 (1986) (3) $143-150$.

[2] Marin L. and Lesnic D., Boundary element solution for the Cauchy problem in linear elasticity using singular value decomposition, Comp. Meth. App. Mech. Eng. 29-30 (2002) 3257-3270.

[3] Galybin A.N., An inverse problem of elastostatics in mechanics of composites, Composites Science and Technology 68 (2008) 1188-1197.

[4] Marin L. and Lesnic D., The method of fundamental solutions for the Cauchy problem in two-dimensional linear elasticity, International Journal of Solids and Structures 41 (2004) 3425-3438

[5] Young D.L., Tsai C.C., Chen C.W., Fan C.M., The method of fundamental solutions and condition number analysis for inverse problem of Laplace equation, Computers and Mathematics with Applications 55 (2008) 1189-1200.

[6] Cimetière A., Delvare F., M. Jaoua M. and F. Pons, Solution of the Cauchy problem using iterated Tikhonov regularisation, Inverse Problems 17 (2001) 553-570.

[7] Hon Y.C., Wei, T., Backus-Gilbert algorithm for the Cauchy problem of the Laplace equation, Inverse Problems 17 (2001) 261-271.

[8] Kozlov V.A., Maz'ya V.G. and Fomin A.V., An iterative method for solving the Cauchy problem for elliptic equations, Comput. Meth. Math. Phys. 31 (1991) (1) 45-52.

[9] Ben Belgacem F; El Fekih, H., On Cauchy's problem: a variational Stecklov-Poincaré theory, Inverse Problems 21 (2006) 1925-1936.

[10] Azaiez M. , Ben Belgacem F;, El Fekih H., On Cauchy's problems : Completion, regularization and approximation, Inverse Problems 22 (2007) 1307-1336.

[11] Knowles I., A variational algorithm for electrical impedance tomography, Inverse Problems 14 (1998) 1513-1525.

[12] Kohn R.V., Mc Kenney A., Numerical implementation of a variational method for electric impedance tomography, Inverse Problems 6 (1990) 389-414.

[13] Ladevèze $\mathrm{P}$ and Leguillon $\mathrm{D}$, Error estimates procedures in the finite element method and applications, SIAM J. Numer. Anal. 20 (1983) 485-509

[14] Ladevèze $\mathrm{P}$ and Chouaki $\mathrm{A}$, Application of a posteriori error estimation for structural model updating, Inverse Problems 15 (1990) 49-58

[15] Ladevèze P., Nonlinear Structural Mechanics-New Approaches and Non-Incremental Methods of Calculation, Springer-Verlag, New York, 1998.

[16] Andrieux S., Baranger T.N., An energy error-based method for the resolution of the Cauchy problem in 3D linear elasticity. Comput. Meth. Appl. Mech. Engrg, 197 (2008) (9-12) 902-920.

[17] Baranger T.N., Andrieux S., An optimization approach for the Cauchy problem in linear elasticity, J. Multidisciplinary optimization 35 (2008) 141-152.

[18] Andrieux S., Baranger T.N. and Ben Abda A., Solving Cauchy problem by minimizing an energy-like functional, Inverse Problems 22 (2006) 115-133.

[19] Escriva X., Baranger T.N., Hariga Tlatli N, Leak identification in porous media by solving the Cauchy problem, C.R. Mécanique 335 (2007) 410-406. 
[20] Hariga-Tlatli N., Baranger T. N., Ben Abda A. and Erhel J., An Inverse Problem in ElectroCardioGraphy, Third conference on Inverse Problems, Control and Shape Optimization. Nice, April 5-7, 2006.

[21] Ragab A. R. and Bayoumi S. E., Engineering solid mechanics: Fundamentals and Applications, CRC Press, 1998.

[22] Bui H.D., Fractures Mechanics, Inverse Problems ans Solutions, Springer, 2006.

[23] Andrieux S., Baranger T.N., Energy error based numerical algorithms for Cauchy problems for nonlinear elliptic or time dependent operators, Inverse Problems Symposium, May 31 to June 2, 2009 Michigan State University, 2009.

[24] Andrieux S., Baranger T.N., Energy method for Cauchy problems of evolutions equations, $6^{\text {th }}$ International Conference on Inverse Problems in Engineering: Theory and Practice, Dourdan (Paris), France - June 15-19, 2008.

[25] Baranger T.N., Andrieux S., Energy method for boundary conditions identification for dynamic problems., 8th. World Congress on Computational Mechanics (WCCM8), 5th. European Congress on Computational Methods in Applied Sciences and Engineering (ECCOMAS 2008), June 30 - July 5, 2008, Venice, Italy.

[26] Code-Aster, 2008, EDF, Clamart, France. www.Code-Aster.org.

[27] Comsol, 2007, Comsol Multiphysics. www.comsol.fr.

Received July 17, 2009

\section{BỔ SUNG DỮ LIỆU CHO CÁC TOÁN TỬ TUYẾN TÍNH ĐỐI XỨNG CŨNG NHƯ BÀI TOÁN CAUCHY: MộT PHƯƠNG PHÁP HIỆU QUẢ DỰA TRÊN SỰ CỰC TIỂU HÓA SAI SỐ DẠNG NĂNG LƯợNG}

Bổ sung dữ liệu là một bài toán trong đó dữ liệu dư đo được hoặc biết được trên một phần biên của miền, trong khi dữ liệu trên phần biên còn lại là không biết. Do đó mục đích là xác định lời giải của phương trình đạo hàm riêng xác định trên toàn miền, thỏa mãn các dữ liệu dư và sau đó xác định những dữ liệu thiếu. Với các toán tử tuyến tính đối xứng, chúng tôi đề xuất một phương pháp tổng quát để giải bài toán bổ sung dữ liệu cũng như bài toán Cauchy. Nhiều ứng dụng khác nhau được mô tả cho các bài toán dẫn (nhiệt điện) ổn định và bài toán đàn hồi tĩnh. 